\title{
More carbon and less salt
}

\author{
We take a closer look at recent developments in research on various strategies to use carbon \\ nanostructures for water desalination.
}

The increasing demand for fresh water for drinking and agriculture is one of the grand challenges of our age. Not only is the global population increasing in numbers and needs, but global warming will have drastic effects on freshwater resources. Facing the challenge will require advances in technology for water remediation and distribution in combination with changes in behaviour to reduce usage and waste.

Still, over two-thirds of the Earth is covered by seawater, from which fresh water can be obtained by removing salt. Desalination is already a major source of fresh water, primarily in dry regions like Australia, Saudi Arabia and California, although desalination plants are also part of the clean water supply in countries with an apparent wealth of resource. The Thames Water Desalination Plant near London for example provides drinking water to almost a million people.

Unfortunately, desalination is not efficient, nor sustainable, at least at present. Reverse osmosis, which is one of the most widely used techniques and arguably among the most-efficient still requires a few kilowatt hours per cubic metre of water produced, which is an order of magnitude larger than the energy needed for the transport and distribution of fresh water. Improving reverse osmosis and finding alternative, moreefficient techniques is therefore paramount.

Engineered nanomaterials have the potential to improve current desalination techniques. In this issue we take a closer look at some of the possibilities offered by carbon nanomaterials like graphene and carbon nanotubes, which in light of their structural and electronic properties are currently studied for the production of more-efficient filtration membranes for reverse osmosis, but also for their use in alternative desalination techniques.

The Review by Rohit Karnik and co-workers (page 509) focuses on the transport mechanism through artificially drilled nanopores in atomic membranes primarily, though not exclusively, graphene based. Although the article is mainly about transport properties at a fundamental level, the authors also consider potential applications. In particular, their high permanence could yield substantial improvement in the energy consumption of reverse osmosis with respect to currently used polyamide membranes.

An Article by Rahul Nair and co-workers (page 546) explores a different mechanism for salt removal. In their work, graphene oxide layers are used to create a sieve. The water does not flow through artificial pores but through two-dimensional capillaries created between the layers. In general, the problem of such a structure is that it swells when immersed in water, inducing a minimum distance between the layers which is larger than diameter of the ions in common salts. Nair and co-authors manage to control the distance by simply encapsulating the graphene oxide layers in epoxy films and effectively force the spacing to a desired value. Their results show that ion permeation rates through these capillaries decrease exponentially with decreasing the capillary size whereas water transport is only weakly affected.

\section{Engineered nanomaterials have the potential to improve current desalination techniques.}

Finally, the work by David Jassby and co-workers (page 557) explores the use carbon nanotubes to improve the performance of membranes used in the desalination process known as membrane distillation. This technique is mainly used for high-salinity brines and is based on the generation of a thermal gradient across a hydrophobic membrane. The gradient is usually achieved by heating the bulk feed solution, but in this work, Jassby and co-authors combined carbon nanotubes with polymers to realize a porous membrane that can also act as the Joule heater, which delivered heat directly to the membrane/water interface. Joule heating produced by carbon-based electrodes immersed in a brine must be protected from electrocorrosion. They demonstrate that under the right conditions of operation the membrane can resist corrosion, and has a recovery efficiency that is greater than what can be achieved with standard membrane distillation.

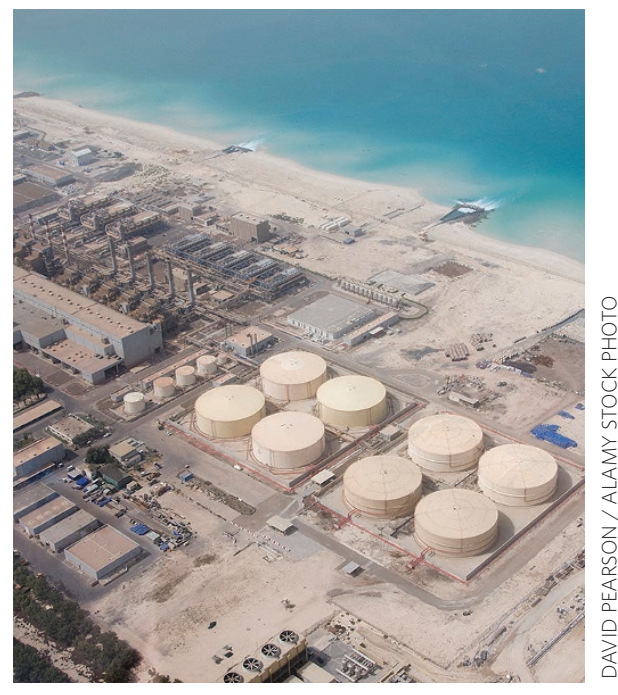

Desalination plant in Dubai.

The articles in this issue illustrate only some of the approaches for the use of carbon nanostructures in desalination. Another interesting approach is the use of graphene oxide materials to enhance absorption in solar desalination, as reported by Jia Zhu and co-workers ${ }^{1,2}$ and more recently by Liangti $\mathrm{Qu}$ and co-authors ${ }^{3}$, which could result in the development of portable water treatment devices.

How far are we from actual implementation of any of these techniques? Perhaps the closest to applications are those in which the nanostructures are used to improve and optimize existing technologies. For example, according to David Jassby, the production of large quantities of the type of carbon nanotubes used in his composite membranes has already been demonstrated. More generally however, as is often the case with nanomaterial-based technologies, the main challenge remains the need to produce large-enough, high-quality material necessary to scale up the devices. 